\title{
The New Algorithm for Fast Probabilistic Hypocenter Locations
}

\author{
Wojciech DĘBSKI and Piotr KLEJMENT
}

Institute of Geophysics, Polish Academy of Sciences, Warsaw, Poland

\begin{abstract}
The spatial location of sources of seismic waves is one of the first tasks when transient waves from natural (uncontrolled) sources are analysed in many branches of physics, including seismology, oceanology, to name a few. It is well recognised that there is no single universal location algorithm which performs equally well in all situations. Source activity and its spatial variability in time, the geometry of recording network, the complexity and heterogeneity of wave velocity distribution are all factors influencing the performance of location algorithms. In this paper we propose a new location algorithm which exploits the reciprocity and time-inverse invariance property of the wave equation. Basing on these symmetries and using a modern finite-difference-type eikonal solver, we have developed a new very fast algorithm performing the full probabilistic (Bayesian) source location. We illustrate an efficiency of the algorithm performing an advanced error analysis for 1647 seismic events from the Rudna copper mine operating in southwestern Poland.
\end{abstract}

Key words: hypocenter location, probabilistic inverse theory, error analysis, time reversal mirroring, numerical methods.

\section{INTRODUCTION}

Determining the spatial location (hypocenter) and origin time of the source of seismic waves is one of the first tasks undertaken when the waves from un- 
controlled sources are analyzed (Aki and Richards 1985, Gibowicz and Kijko 1994). Depending on the type of event, different location techniques are used. If recorded waveforms for the event at hand exhibit well defined and sharp waves onsets, the event is usually located by seeking a point in space for which numerically predicted wave onset times fit the observed ones (Aki and Richards 1985, Bulland 1976, Thurber and Rabinowitz 2000). This can be accomplished by any optimization technique (Aki and Richards 1985, Thurber and Rabinowitz 2000). The price for simplicity and numerical efficiency of such approach is, however, a lack of reliable estimation of the location errors. Within this optimization-based approach, the most popular method of error evaluation is a linearization of the forward modelling procedure around the optimum location found and calculation of the covariance matrix (Husen and Hardebeck 2010, Menke 1989, Pavlis 1992). The diagonal elements are then interpreted as squares of the location errors for each coordinate, respectively, and the "horizontal part" of the covariance matrix gives a nice looking "error ellipse" confidence region - a region around the solution found where the epicenter is supposed to be located within obtained errors. However, as it has been pointed out, for example, by Bai et al. (2006), Husen et al. (1999), Lomax et al. (2001), Wiejacz and Debski (2001), in realistic situations this estimation of location errors is too simplified and often unrealistic. The reason is that the cornerstone of the method linearization of the forward modelling relation leads to a quadratic misfit function which is a good choice only when all uncertainties pertaining location procedure (data errors, modelling errors) are Gaussian. This is often not the case (Husen and Hardebeck 2010, Lomax et al. 2009, Rudzinski and Debski 2012).

To evaluate the location errors in a more systematic way, a probabilistic, also called Bayesian, inversion technique must be used (Tarantola 2005). The approach relies on exploring the space of all possible source locations and assigning to each point in this space (i.e., each possible location) a probability of being the true hypocenter location (Debski 2010, Sambridge and Mosegaard 2002, Tarantola 2005). This a posteriori probability can then be used for any analysis of location errors using standard statistical methods (Debski 2010, Lehmann and Casella 1998).

For completness it is worth mentioning the third method of error estimation which is based on the direct Monte Carlo simulation (Giovambattista and Barba 1997, Husen and Hardebeck 2010). The method relies on adding the Monte Carlo generated "noise" to the synthetic data calculated for the formerly found optimum location and relocating the event using these "noisy" data. The size of a "cloud" of the obtained solutions is used as an estimator of the location errors. However, as pointed out by Debski (2004), this approach reduces to the above-mentioned probabilistic approach if the "noise" is generated according to the probability distribution of joint modeling and observational errors. 
The probabilistic approach to hypocenter location provides the most complete information about the solution found (see, e.g., Lomax et al. 2009, Tarantola 2005). However, the method is computationally demanding as it requires an exhaustive exploration of the 3 or 4-dimensional model space. Even the use of modern, efficient Markov Chain Monte Carlo based methods (Chib and Greenberg 1995, Debski 2010, Gilks et al. 1995, Lomax et al. 2009) does not allow to employ this approach in applications requiring prompt results. The new perspective for using probabilistic approach are opened by using the time reversal and reciprocity principles of the wave equation together with finite-difference type forward modelling solvers. In this case, as we shall discuss latter on, one can avoid an explicit sampling of the a posteriori distribution - the most time consuming part of the probabilistic inversion requiring tens of thousends forward modellings by performing an "implicit" sampling through an examination of the a posteriori distribution over the finite grid used by the forward modelling solver. This reduces a number of required forward modelings from thousands to only a few - a number of used seismic stations. In this paper we descibe such an algorithm. We call it TRMLOC.

The paper is structured as follow. Firstly, after a short description of the source location task the proposed algorithm is described. Next, two basic elements of the algorithm, namely probabilistic inversion technique and the FSM algorithm are discussed. Next, the algorithm is compared to other popular methods and its performance is illustrated by analyzing 1647 mining induced seismic events from Rudna (Poland) deep copper mine. The conclusions and general discussion ends up the paper.

\section{LOCATION ALGORITHMS}

Let us assume that to locate a given source the arrival times $\mathbf{t}_{i}^{\text {obs }} i=1 \cdots N_{s}$ are read from waveforms recorded by $N_{s}$ sensors (geophones, seismometers, piezoceramic transducers, etc.). Let $\mathbf{t}_{i}^{t h}(\mathbf{m})$ denote the theoretically predicted arrival time of waves originating at the point $\mathbf{m}$ and recorded by $i^{\text {th }}$ sensor where the location parameters $\mathbf{m}=\left(\vec{r}, t_{o}\right)$ include three spatial coordinates $(\vec{r}=(x, y, z))$ and the origin time of the event $t_{o}$, so

$$
\mathbf{t}_{i}^{t h}(\mathbf{m})=t_{o}+\Delta_{i}(\vec{r})
$$

where $\Delta_{i}(\vec{r})$ is the propagation time from the source to a given sensor.

Finding the hypocenter location can now be formulated as the optimization task (Aki and Richards 1985): searching for the model $\mathbf{m}^{m l}$ which minimizes the differences between observed ( $\mathbf{t}_{i}^{\text {obs }}$ ) and predicted $\left(\mathbf{t}_{i}^{\text {th }}(\mathbf{m})\right)$ travel times. The solution can be obtained by means of any convenient numerical optimization algorithm. 
To complete the location task, an error analysis should be performed in order to evaluate the reliability of the solution. The most popular approach to this task is based on the linearizion of the optimized function $S(\mathbf{m})$ around the solution $\mathbf{m}^{m l}$ and calculation of the covariance matrix (Gibowicz and Kijko 1994). However, this simple method is often not reliable. It fails if observational and/or modeling errors have nontrivial statistics, the recording network geometry is complicated or the velocity model has a complex structure (Lomax 2005, Lomax et al. 2009, Rudzinski and Debski 2012).

Another, probabilistic approach to the source location task relies on assigning to each model $\mathbf{m}$ (point in space and time) the a posteriori probability of m being the true source location (Debski 2010, Lomax et al. 2009, 2000). The advantage of this approach is the possibility of full and exhaustive error and resolution analysis (Debski 2010). In the simplest case, the a posteriori probability density $\sigma(\mathbf{m})$ assigned to model $\mathbf{m}$ reads (Debski 2010, Mosegaard and Tarantola 2002)

$$
\sigma(\mathbf{m})=\frac{1}{Z} f(\mathbf{m}) L(\mathbf{m})
$$

where $Z$ is the normalization factor called evidence, $f(\mathbf{m})$ is the probability density function describing the a priori estimation of the source location. The second term, traditionally called the likelihood function, is defined as follows

$$
L(\mathbf{m})=\exp (-S(\mathbf{m}))
$$

where

$$
S(\mathbf{m})=\left\|\mathbf{t}_{i}^{t h}(\mathbf{m})-\mathbf{t}_{i}^{o b s}\right\|
$$

is the so-called misfit function and $\|\cdot\|$ is a norm in the data space. The choice of a given norm $\left(l_{1}, l_{2}\right.$, Cauchy, etc.) reflects our expectations about errors statistics, existence of outliers, systematic bias, etc (Debski 2010).

Various numerical estimators, like the maximum likelihood model $\left(\mathbf{m}^{m l}\right)$ which maximizes $\sigma(\mathbf{m})$, the average model $\left(\mathbf{m}^{a v r}\right)$, the covariance matrix, etc. can easily be calculated from $\sigma(\mathbf{m})$. The technique is very general but it requires exhaustive sampling of the model space to determine the characteristics of $\sigma(\mathbf{m})$. Consequently, the approach is computationally demanding even if the very efficient Markov Chain Monte Carlo sampling technique (Debski 2010, Gilks et al. 1995, Lomax et al. 2009) is used.

The new possibilities of the full probabilistic seismic data inversion for hypocenter coordinates open when the time reversal mirroring technique is employed. This technique, carefully analyzed in laboratory experiments (see, e.g., Fink 1997, Ulrich et al. 2008), by numerical simulations (see, e.g., Kremers et al. 2011, Scalerandi et al. 2009, Steiner and Saenger 2012), and theoretical investigations (see, e.g., Masson et al. 2014, Tromp et al. 2005, Ulrich et al. 2009) has already found application in seismic prospecting (see, e.g., Gajewski 
and Tessmer 2010, Witten and Artman 2011) and location of seismic tremors (see, e.g., Artman et al. 2010, Larmat et al. 2008, O'Brien et al. 2011). Combaining this technique with a modern eikonal solver has lead us to the proposition of the new TRMLOC location algorithm which enables very fast Bayesian inversion of travel time onset data for hypocenter location.

\section{TRMLOC ALGORITHM}

The wave equation (Aki and Richards 1985) describing the propagation of seismic waves exhibits two very important features. First of all, being a secondorder partial differential equation with even time derivatives, the equation is invariant under time reversal. Thus, if only the boundary conditions do not depend on time, the solutions for forward and back propagation of waves in time are identical. Secondly, the equation exhibits spatial reciprocity invariance, which means that wave propagation between two arbitrary points is invariant with respect to the exchange of these points: the seismogram from the source located at point $A$ and recorded at point $B$ is the same as the seismogram from the source set at $\mathrm{B}$ and recorded at $\mathrm{A}$. Combining both properties of the wave equation has enabled construction of a very simple and efficient numerical algorithm used for an analysis presented in this paper. It relies on putting the virtual sources at the receiver locations (reciprocity principle) and simulating propagation of seismic waves from such virtual sources adopting the recorded real signals with reversed time as their temporal signature: the last arriving signal is "re-sent" as the first one. Due to complicated interference, the re-sent signals not only focus in the point where the real source ruptured and at the origin time of the rupture but also provide very important information on the kinematics and dynamics of the rupture process (Fink et al. 2000, Fink and Tanter 2010, Kremers et al. 2011).

The existing application of the above-described time reversal technique for hypocenter location consists of two steps, namely back propagation ("resending") of recorded signals and scanning of the model (location) space in order to find the optimum location where all the back-propagated signals positively interfere (O`Brien et al. 2011). The algorithm used in this paper extends the above idea in two aspects. First of all, instead of searching for a point at which the largest positive interference occurs, we propose to construct the $a$ posteriori probability density based on the differences of the back-propagated observational data. Secondly, for the location of sources with well determined time onsets on a given set of sensors, we do not need to perform a full waveform back propagation. Instead, we can consider only the wavefronts whose propagation in time is described by the much simpler to solve eikonal equation (Aki and Richards 1985) for which modern fast algorithms like the Fast Sweeping Method (Zhao 2005), the Fast Marching Method (Sethian 1999) or the more tra- 
ditional finite difference approaches (Podvin and Lacomte 1991, Vidale 1990) can be used.

We propose to construct the a posteriori probability density using the differential misfit function $\bar{S}(\mathbf{m})$

$$
\bar{S}(\mathbf{m})=\frac{1}{2 N_{s}} \sum_{i, k ; i \neq k}\left\|\mathbf{t}_{m}^{i}-\mathbf{t}_{m}^{k}\right\|
$$

based on the difference of the back propagated wavefronts from all considered sensors. In this equation, $\mathbf{t}_{m}^{i}$ and $\mathbf{t}_{m}^{k}$ stand for waveform onsets recorded by $i$-th and $k$-th receivers, respectively, and back-propagated to the point $\mathbf{m}$. $N_{s}$ is the number of the receivers used (number of available observational data) and $1 / 2$ takes into account the symmetry of the sum. Following this assumption, we postulate the a posteriori probability distribution as

$$
\sigma(\mathbf{m})=\operatorname{const.} f(\mathbf{m}) \exp (-\bar{S}(\mathbf{m}))
$$

The physical intuition behind the above definition of $S(\mathbf{m})$ is quite clear. In an ideal case (no noise, exact forward modelling) all back-propagated travel times should be equal to the source origin time $\left(t_{o}\right)$ at the true hypocenter location point. Thus, the condition for the hypocenter location is the equality of all back-propagated arrival times. Due to the presences of observational and modeling errors, this condition cannot in general be directly fulfilled and thus a reasonable solution is to look for the point in space where $S(\mathbf{m})$ gets minimum. Let us note that, as follows from Eq. 1, the origin time $t_{o}$ does not enter $S(\mathbf{m})$ and thus an original 4D inversion is reduced to 3D problem: a search for the hypocenter's spatial coordinates only.

The idea of using the differential-time form of the misfit function is by no means new and can be traced back in time to Zhou (1994) and Matsu'ura (1984). In various forms it has already been implemented in different optimizationbased location algorithms under various names, among which the equal difference time (EDT) is the most popular (see, e.g., Font et al. 2004, Lomax et al. 2009, Zhou 1994). The EDT formulation relies on searching the point $\mathbf{m}$ for which hyperbolic surfaces defined by the condition

$$
\Delta_{i}(\mathbf{m})-\Delta_{j}(\mathbf{m})=\mathbf{t}_{i}^{o b s}-\mathbf{t}_{j}^{o b s}
$$

intersect for all pairs of stations $(i, j)$. This condition can be rewritten as $\mathbf{t}_{i}^{\text {obs }}$ $\Delta_{i}(\mathbf{m})=\mathbf{t}_{j}^{o b s}-\Delta_{j}(\mathbf{m})$ for all $(i, j)$, which is actually the condition of equality of all back-propagated observational time onsets at the hypocenter location. The advantage of using the EDT-type differential misfit function relies in removing of origin time from inversion procedure (Matsu'ura 1984) and also lower sensitivity of location results to velocity model (Font et al. 2004, Rudzinski and 
Debski 2011, Waldhauser and Ellsworth 2000, Zhou 1994). Additionally, in the developed algorithm, it has also allowed to perform an implicit sampling of the a posteriori distribution, as discussed latter on. The EDT type misfit function is the cornerstone of the modern relative location methods, namely the double differences and extended double differences techniques (Rudzinski and Debski 2012, Waldhauser and Ellsworth 2000).

Finding the minimum of $S(\mathbf{m})$ will provide the hypocenter location, so $S(\mathbf{m})$ can serve as the cost function for any optimization-based location algorithm. Much less obvious is whether this misfit function can also be used within the probabilistic inversion framework for generating the likelihood function according to Eq. 6 . The problem is that the "true" likelihood function $L(\mathbf{m})$ defined by the probabilistic inverse theory is actually a convolution of probability distributions of observational and modelling errors (Debski 2010, Tarantola 2005). Thus, from the statistical point of view, it describes the statistic of sum of errors. Apparently the function $L(\mathbf{m})$ defined by $\bar{S}(\mathbf{m})$ is not such a statistic. It is rather the statistic of sum of differential errors so the question is if the errors estimated by using this proxy of the likelihood function are not systematically biased. Although this point has not been clarified yet, the differential misfit function has already been implemented in some probabilistic location algorithms (Lomax et al. 2000, Rudzinski and Debski 2011) and we use it also in the TRMLOC algorithm.

Having defined the a posteriori distribution $\sigma(\mathbf{m})$ we have to explore the space of model parameters in order to obtain various characteristics of $\sigma(\mathbf{m})$ including the position of the global maximum, checking an existence of secondary maxima, etc. This is the most demanding numerical part of any probabilistic inversion. However, in case of the location task the model space which has to be sampled is exactly the same space (3D configuration space) as that over which the forward modelling operator acts. This opens a possibility of performing an implicit sampling of the a posteriori distribution simultaneously with solving the forward problem. The idea is as follows. Assume that the forward modeling method used to calculate $\Delta_{i}(\mathbf{m})$ is able to provide the back-in-time propagated observed time onsets for a set (for example, regular grided) of spatial points. Then, according to Eq. 5, the a posteriori distribution $\sigma(\mathbf{m})$ can be immediately calculated with minimum numerical computations for all grid points. This way we have sampled $\sigma(\mathbf{m})$ at all these points. If the points form a dense enough, regular set, we end up with the well sampled $\sigma(\mathbf{m})$ so we do not need any additional sampling indispensable in the classical probabilistic inversion. We call this mechanism the implicit sampling. The forward modeling techniques fulfilling the above requirement are the all wave equation or eikonal solvers based on the finite difference, finite element, spectral elements, or similar numerical methods (Sethian 1999, Virieux et al. 2009). Thus, summarizing the 
- Discretize space $\mathbf{m}=\left(X_{i}, Y_{j}, Z_{k}\right), \quad i, j, k=1,2, \ldots$

- Set the a priori density function $f(\mathbf{m})$

- Repeat for each receiver (in parallel)

- back propagate observed time onsets $\mathbf{t}_{i}^{o b s}$ using eikonal (FSM) solver on defined spatial grid

- Calculate $S(\mathbf{m})=\frac{1}{2 N_{s}} \sum_{i, k ; i \neq k}\left(\mathbf{t}_{m}^{i}-\mathbf{t}_{m}^{k}\right)^{2} / C_{p}^{2}$

- Determine $\sigma(\mathbf{m})=$ const. $f(\mathbf{m}) \exp (-S(\mathbf{m}))$

- Calculate statistical estimators $\mathbf{m}^{m l}, \mathbf{m}^{a v r}, \Delta \mathbf{m}$, evidence, entropy, etc.

- If needed, perform inspection of the full $\sigma(\mathbf{m})$ or marginal a posteriori distributions

Fig. 1. The basic steps of the TRMLOC algorithm.

above consideration we propose the algorithm whose flowchart is shown in Fig. 1.

One very important feature of the algorithm is its high speed, as will be demonstrated later on, which follows from:

- reducing inverse problem from 4D to 3D by eliminating event's origin time from inversion,

- employing the modern finite-difference very fast eikonal solver,

- avoiding explicit sampling of the model space: $\sigma(\mathbf{m})$ is evaluated at each grid nodes simultaneously with forward modelings,

- parallelization of the algorithm.

Let us also note that the backward propagation of the observed time onsets through the back-in-time forward modeling has to be performed only $N_{s}$ times - as many as the number of sensors is used. This is a direct advantage of using the time-reversal and reciprocity invariance principle.

Actually, the TRMLOC algorithm is very similar to the algorithm NLloc developed by Lomax et al. (2000). Both approaches use the probabilistic inverse approach, eikonal solvers for forward modelling and similar EDT-based likelihood function. The main differences arise from using different eikonal solvers (NLloc uses the method of Podvin and Lacomte (1991) while TRMLOC the Fast Sweeping Method) and from different implementation of the a posteriori pdf sampler.

The very important element of the TRMLOC algorithm is the eikonal solver which enables very efficient calculation of the wavefront positions in the entire 3D domain for a general velocity model. Constructing the TRMLOC algorithm, two finite-difference type eikonal solvers were considered, namely the Fast Marching Method (FMA) developed by Sethian (1999) and the Fast Sweeping Method (FSM) developed by Zhao (2005). The FMA algorithm exhibits 
numerical complexity of the order of $N \log (N)$, where $N$ is the number of all grid nodes and is optimal for complex velocity models (Sethian 1999). The FSM method is faster for smooth velocity models with numerical complexity proportional to $N$ but it is over-performed by FMA in cases of complex velocity models. TRMLOC has been designed for a local/regional analysis when velocity models are relatively smooth so the FSM technique has been selected. Since this algorithm is practically unknown to seismological community we give here its detailed description restricting ourselves to $2 \mathrm{D}$ case for the sake of compactness.

\subsection{Eikonal solver: Fast Sweeping Method}

Under the high frequency approximation the full wave equation can be split into the eikonal equation describing spatial propagation of wavefronts and transport equation describing changes of wave amplitudes. As we are interested here in travel times modeling, we consider only the eikonal equation which together with the boundary condition at source location $\Gamma$ reads

$$
\begin{aligned}
& \nabla T \cdot \nabla T=\frac{1}{v^{2}} \\
& \left.T\right|_{\Gamma}=0
\end{aligned}
$$

where $T$ describes wavefront position in space originating from the source at $\Gamma$ and $v$ denotes velocity. This is a special case of the Hamiltonian-Jacobi, hyperbolic type nonlinear equation for which the term on right-hand side is always positive. For numerical purpose, such equation can be discretized by using the first-order Godunov upwind type discretization (Sethian 1999, Zhao 2005), For internal grid points this finite difference scheme leads to the following discrete approximation of Eq. 8

$$
\left[\left(T_{i, j}-T_{x m i n}\right)^{+}\right]^{2}+\left[\left(T_{i, j}-T_{y m i n}\right)^{+}\right]^{2}=h^{2} s_{i j}^{2}
$$

where $i, j$ are indexes of the grid point $\mathbf{x}_{i, j}=\left(x_{i}, y_{j}\right), h$ is the grid size (for simplicity, the quadratic grid is assumed), $s_{i j}$ is the value of slowness at grid point $\left(\mathbf{x}_{i, j}\right)\left(s_{i j}=1 / v_{i j}\right)$, and the following shorthand notation is used:

$$
T_{x \min }=\min \left(T_{i-1, j}, T_{i+1, j}\right), \quad T_{y \min }=\min \left(T_{i, j-1}, T_{i, j+1}\right)
$$

and

$$
(x)^{+}=\left\{\begin{array}{l}
x, x>0 \\
0, x \leq 0
\end{array}\right.
$$

The Fast Sweeping Algorithm proposed by Zhao (2005) is using the above discretization and solves the resulting system of nonlinear equation iteratively as follows: 
- Initialization: a large positive value is assigned to all $T_{i, j}$. Then, for all grid points $\left(\mathbf{x}_{i, j}^{s}\right)$ within the source of waves (it can be a single grid node for a point-like seismic source model or an extended area $\Gamma$ for the finite source model) the boundary condition $T\left(\mathbf{x}^{s}\right)=0$ is set.

- Iterations with alternating sweeping: the following procedure is repeated until the conversion to stable solution is reached.

- At each grid point $\left(\mathbf{x}_{i, j}\right)$ not set during the initializations the solution $\tilde{T}$ is computed using current values of $T$ at neighborhood points and then $T_{i, j}$ is updated us follows

$$
T_{i, j}^{\text {new }}=\min \left(T_{i, j}^{c u r}, \tilde{T}\right)
$$

where the updating solution $\tilde{T}$ is the solution of Eq. 9 and reads

$$
\tilde{T}_{i, j}= \begin{cases}\min \left(T_{x \min }, T_{y \min }\right)+s_{i j} h & \left|T_{x \min }-T_{y \min }\right| \geq s_{i j} h, \\ \frac{T_{x \min }+T_{y \min }+\sqrt{2 s_{i j}^{2} h^{2}-\left(T_{x \min }-T_{y \min }\right)^{2}}}{2} & \left|T_{x \min }-T_{y \min }\right|<s_{i j} h,\end{cases}
$$

- During one iteration the value of $\tilde{T}$ is recalculated four times with different alternating orderings of grid sweeping:
a) $i=1: N_{x}, j=1: N_{y}$
b) $i=1: N_{x}, j=N_{y}: 1$
c) $i=N_{x}: 1, j=1: N_{y}$
d) $i=N_{x}: 1, j=N_{y}: 1$

As follows from the above description, the proposed scheme shows the numerical complexity of order $O(k N)$ where $N$ is the total number of the grid nodes and $k$ is a constant depending on the number of iterations.

The number of iterations to be performed depends on the complexity of the velocity model. In many cases, if the velocity model is reasonably smooth and without large velocity contrasts only a few (usually 2-3) iterations are sufficient for convergence of the algorithm. The reason is that each sweep (Eq. 14) provides the exact solution in one iteration for one spatial quarter, provided the characteristics of the eikonal equation do not intersect (Zhao 2005). This is the case of smooth velocity models. Moreover, the upwind Godunov difference scheme enforces the causality of the solution (Sethian 1999), because the solution at a given grid point is determined by only those neighborhood points for which $T$ is smaller. This is exactly what happens (Heughen's principle) during an advancing of the wavefront. In consequence, the iteration procedure converges very quickly and the solution is optimally accurate. However, we have to 
keep in mind that the used upwind scheme is based on the first order difference stencil. This implies the first order accuracy of the method.

In Fig. 2 an example of a simulation of wavefront positions for the velocity model of Rudna mine (vertical section) is shown. The ability of the algorithm to model the complex wavefront structure, including reflection and refraction effects, is clearly visible. The computation time for this simulation (2D grid with $N=4 \cdot 10^{5}$ grid points) on 4 cores $2.4 \mathrm{GHz}$ clocked Intel processor was about 90 milliseconds.

Being based on general concepts of the modern probabilistic inverse theory, the TRMLOC algorithm exhibits the same level of generality as any other, more traditional Bayesian location algorithms, However, unlike the classical probabilistic approaches, it performs implicit sampling simultaneously with the forward modeling due to the use of the finite difference based eikonal solver and EDT type of the misfit function.

The algorithm has also some limitations. First of all, the eikonal solver provides solutions only for the first arriving seismic phases (or the first arriving $P$ or $S$ waves in case of elastic waves). Including other phases within TRMLOC is possible, but it requires using the full waveform modeling algorithms, or multi-phases extensions to eikonal solver (Hauser et al. 2008, Rawlinson and Sambridge 2004). In both cases, however, the numerical efficiency of the algorithm is lost.
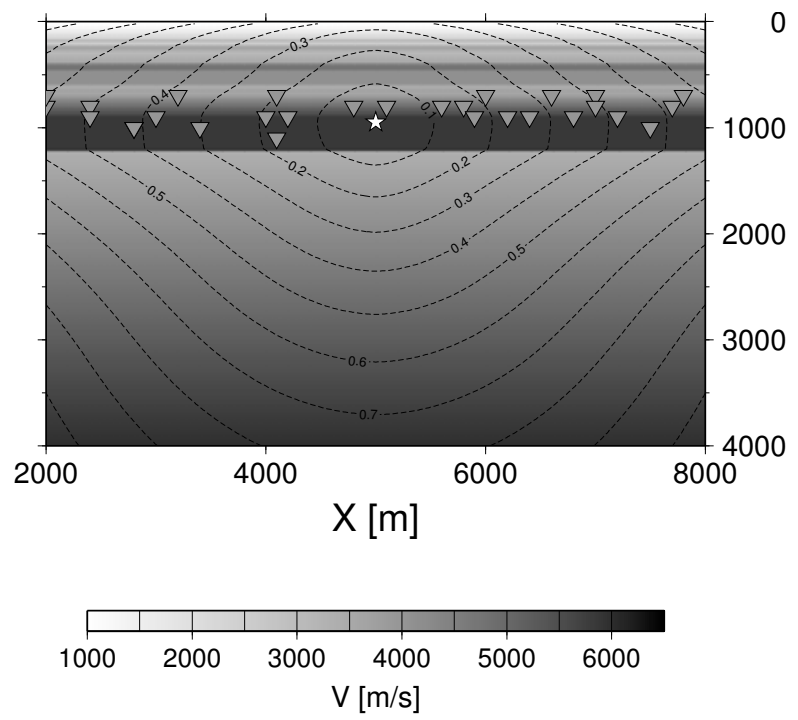

Fig. 2. The vertical section of the synthetic velocity model and wavefront positions simulated by FSM technique from the hypothetic rockburst (star). Open triangles denote seismometers of the underground seismic network operating in the mine. 
The next limitation of the algorithm is related to the fact that the spatial resolution achieved by the algorithm is limited by the grid size used by the forward modeling algorithm. Achieving higher resolution requires a finer spatial grid but this increases the computation time linearly. Another problem connected to the spatial grid is that the eikonal solver used by TRMLOC is the first-order differential solver requiring quite fine grid for high numerical accuracy. Using higher-order solvers or more advanced front propagation techniques (Zhang et al. 2005) may thus be advisable.

An accumulated experience gathered when using the TRMLOC algorithm shows that the most time-critical part of the algorithm is calculation of integrated statistical characteristics of the a posteriori distribution like evidence, entropy, average model, etc. For a large grid, with the number of nodes of order $10^{8}$ it takes about $70-80 \%$ of the whole calculation time. A remedy to this bottle-necked part of the algorithm is its redesigning using GPGPU technology which is extremely efficient in this type calculations (Kloc and Danek 2012). Further efficiency improvement is expected by porting the algorithm, especially the forward modeling part, to the parallel distributed computational platform, using, for example, MPI paralelization schemata (Quinn 2008).

\subsection{Algorithms comparison}

While getting the solution of the location task is conceptually rather simple, at least when the problem is formulated as the optimization task, estimating the reliability of the obtained solution is already more problematic and actually determines an efficiency of all location algorithms. Here we present a simple comparison of some popular algorithms based on counting the number of forward modelings ( $N_{f}$ ) necessary for obtaining the solution and accompanying error estimators. Although such a comparison is not quite fair because different algorithms use different forward modeling techniques (ray tracing, eikonal solver, waveform modeling, etc.) it well illustrates the general feature, namely overall calculation times for different algorithms. In Table 1 we summarize such information for some most popular location softwares.

The first raw in Table 1 lists the most popular location softwares which solve the location tasks through the optimization procedure. The underlying algorithms are very fast, especially due to the fact that they use fast ray-tracers for point-to-point forward modelling. However, the reliability of the estimated location errors can be questioned, especially in complex velocity settings. The second listed group represents algorithms performing probabilistic location. All of them provide possibilities of an exhaustive error analysis, but in general are slower than those from the previous groups. 
Comparison of a number of forward modelings $N_{F}$

performed by selected location algorithms

\begin{tabular}{|l|l|l|l|c|}
\hline \multicolumn{1}{|c|}{ Method } & \multicolumn{1}{|c|}{ Implementation } & \multicolumn{1}{c|}{ Modeling } & \multicolumn{1}{c|}{ Sampling } & $N_{F}$ \\
\hline Linearized & Hypo71(A) $_{\text {iterative }}$ & ray tracer & - & $K \times N_{s}$ \\
inversion & HYPOELLIPSE $^{(B)}$ & ray tracer & - & $K \times N_{s}$ \\
Probabilistic & Rloc $^{(\mathrm{E})}$ & ray tracer & - & $K \times N_{s}$ \\
inversion & NLloc $^{(\mathrm{D})}$ & ray tracer & Metropolis & $\sim 10^{6}$ \\
& TRMLOC & eikonal solver & Gibbs, Oct-Tree & $N_{s}$ \\
& eikonal solver & implicit grid sampling & $N_{s}$ \\
\hline
\end{tabular}

Explanations: A - Lee and Lahr (1975), B - Lahr (1989), C - Klein (2002), D Lomax et al. (2000), E - Rudzinski and Debski (2012). $N_{s}$ stands for number of stations (data) used for hypocenter location and $K$ is a constant which depends on a number of iterations, velocity model, station distributions, etc., and typically ranges between 10 and 50 .

The most interesting is a comparison of NLloc and TRMLOC software as both implement in a different way almost identical location algorithms. The NLloc code provides a large flexibility (two different types of the misfit function and two different sampling methods), which makes it very versatile. On the other hand, TRMLOC is more specialized (mining or local events location) and uses only the EDT type misfit function (with $l_{1}, l_{2}$, and Cauchy norms). The most important difference between NLloc and TRMLOC is that NLloc performs classical, explicit sampling of the location space (3D or 4D) using the modern Oct-Tree or Gibbs samplers. It requires generation and evaluation of $\sim 10^{4}$ or more samples (Lomax et al. 2000) and the a posteriori pdf is then retrieved from the samples distribution. On the other hand, TRMLOC performs implicit exhaustive grid sampling evaluating a posteriori pdf for all grid nodes (typically $\sim 10^{7}$ ) but it is done simultaneously with forward modelling. Computational costs of this implicit sampling are comparable to an additional forward modelling. The other difference is that NLloc saves the forward modelling results on disk and then retrieves it when necessary, while TRMLOC keeps them in the memory. Finally, the TRMLOC algorithm uses the shared memory model of parallel computation via the OMP standard (Dagum and Menon 2002, Quinn 2008) and can easily be ported to MPI and GPGPU parallel computational models, while NLloc (version 6) does not support parallel computations. In consequence of the above implementation differences, the TRMLOC will typically run significantly faster than NLloc on modern computers with multi-core processors and large memories but will be over-performed by NLloc on simpler computers. 


\section{EXAMPLE: RUDNA COPPER MINE}

The TRMLOC algorithm has been applied to locate a set of mining tremors induced by mining activity in the Rudna copper mine. This mine, situated in south-western Poland, runs a digital seismic network composed of 32 vertical seismometers located underground at exploitation depths ranging from 550 to $1150 \mathrm{~m}$. The sampling period is $d t=2 \mathrm{~ms}$. We have analysed 1647 events which occurred in two sections of the mine, between 2012 and 2014. The magnitudes $M_{L}$ of the selected events is ranging from about 1.0 up to 3.5. The histogram of the magnitude distribution of the analysed events is shown in Fig. 3. We have assumed the Gaussian form of the a priori function

$$
f(\mathbf{m})=\exp \left(-\frac{\left(x-x_{a}\right)^{2}+\left(y-y_{a}\right)^{2}}{C_{e}^{2}}+\frac{\left(z-z_{a}\right)^{2}}{C_{z}^{2}}\right)
$$

with $C_{z}=500$ and $C_{e}=2000$ and the a priori solutions $\left(x_{a}, y_{a}, z_{a}\right)$ were provided by the mine. Choosing such values of $C_{z}$ and $C_{e}$ guarantees a very weak a priori constraining of the final solution. The parameter $C_{p}$ defining the likelihood function was taken as $C_{p}=0.01 \mathrm{~s}$. and its setting is discussed in Debski (2015). Let us note at this point that parameters $C_{z}$ and $C_{e}$ quantify the a priori expected location uncertainties with respect to the a priori location $\left(x_{a}, y_{a}, z_{a}\right)$ (Debski 2010). Finally, following the standard mining practice we have used in this preliminary study the constant velocity model assuming P-wave velocity $V=5900 \mathrm{~m} / \mathrm{s}$.

Using the mining data we have firstly verified the scaling property of the TRMLOC algorithm with respect to the number of grid nodes used for calculations. For this analysis we have chosen another event of magnitude 3.6 which occurred on 26 June 2010, and due to its energy was recorded by all 30 running seismometers. Different grid sizes $h$, ranging from 10 up to $200 \mathrm{~m}$, were used

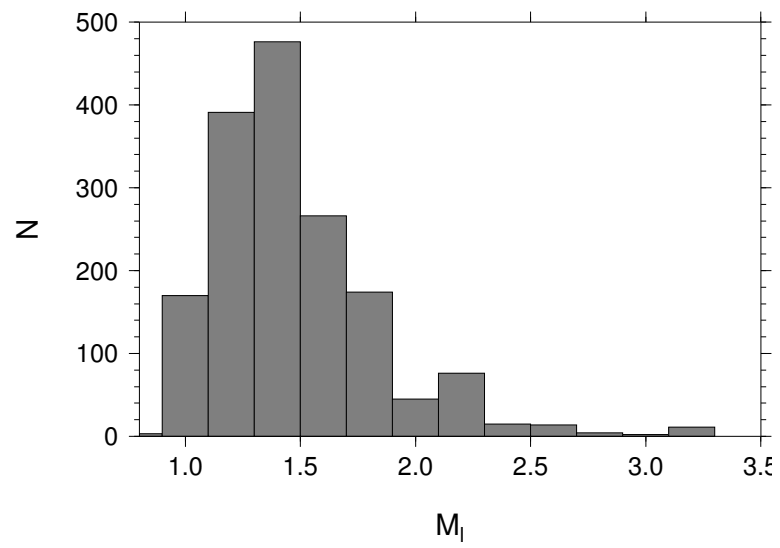

Fig. 3. Magnitude distribution of the analysed 1647 events. 
for data processing, keeping the search region $(\mathrm{X} \times \mathrm{Y} \times \mathrm{Z}) 10 \mathrm{~km} \times 10 \mathrm{~km}$ $\times 2 \mathrm{~km}$ the same for all grid settings. The computational efficiency of the algorithm is determined by a few factors, namely the efficiency of the forward modelling procedure, complexity of calculation of the a posteriori distribution and finally, calculations of various characteristics of the a posteriori distribution. Since numerical efficiency of the FSM solver is $O(N)$ and calculations of the a posteriori distribution and its characteristic is also of order $O(N)$, the expected overall efficiency is also proportional to $N$. The calculations were performed on 8 cores shared-memory computer with $2.4 \mathrm{GHz}$ clock. The calculations were performed 50 times for each grid size to avoid a bias introduced by other tasks performed by operating system and the minimum computational times were recorded. Their dependence on the total number of grid nodes $N$ and the grid size $h$ is shown in Fig. 4. The theoretically predicted linear re-
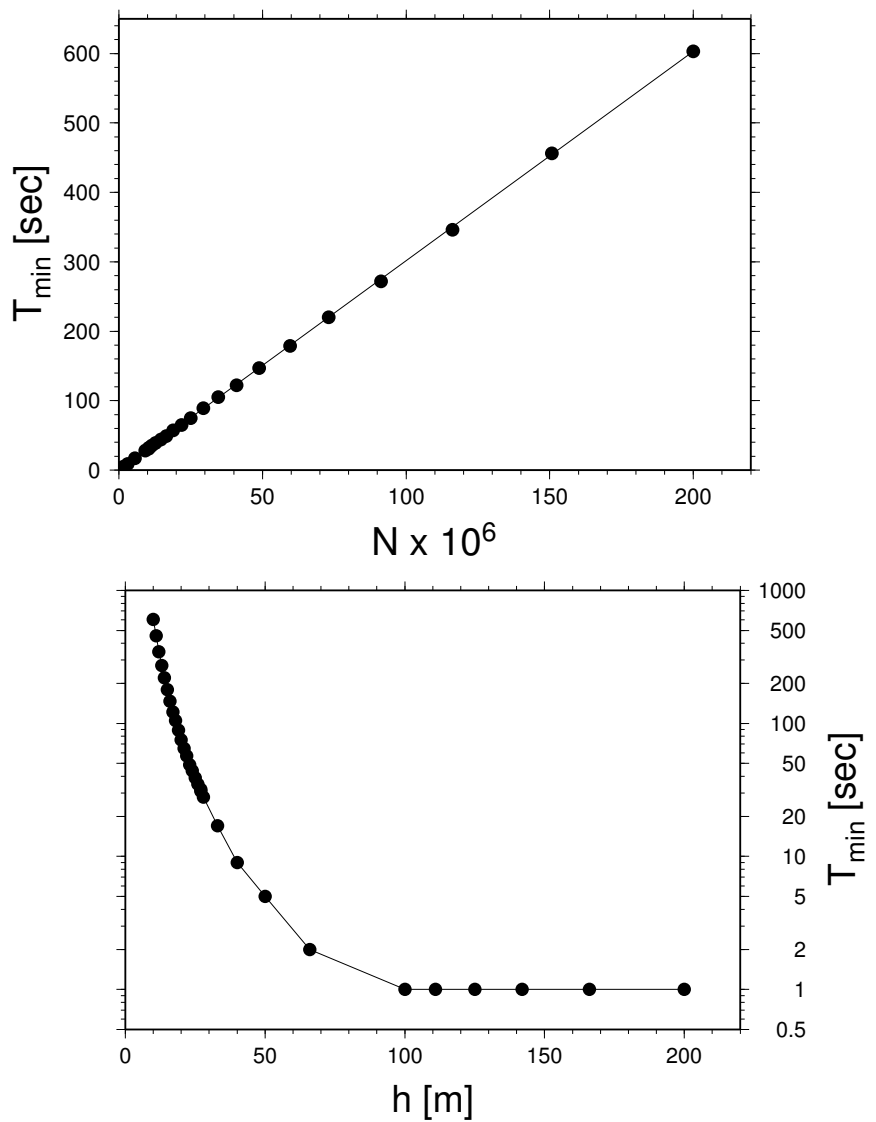

Fig. 4. The TRMLOC minimum computational time taken from an ensemble of 50 runs as a function of number of grid nodes $N$ (up) and grid spacing $h$ (down). Theoretically predicted linear dependence of computational time with $N$ is clearly visible. 
lation between computational time and number of grid nodes is clearly seen. Moreover, for all reasonable grid choices for which grid spacing $h$ is smaller than the desired location accuracy $(20-50 \mathrm{~m})$ the calculation times are about $1 \mathrm{~min}$. This is a fully acceptable computational efficiency which allows to use the algorithm in time critical application like a routine seismic data processing in mines.

In the next step we have analysed the location accuracy estimated by the diagonal elements of the a posteriori covariance matrix. The results are shown in Fig. 5.

It is clearly visible from Fig. 5 that epicentral ( $x$ and $y$ ) coordinates are much better resolved than the vertical one. This well known fact is a consequence of an almost planar geometry of the seismic network operating by mine. The horizontal location accuracy ranging in most cases between 10 and 30 meters is quite satisfactory. In case of the depth component the location accuracy is ranging typically between 100 and $300 \mathrm{~m}$. As we discuss latter, on the largest
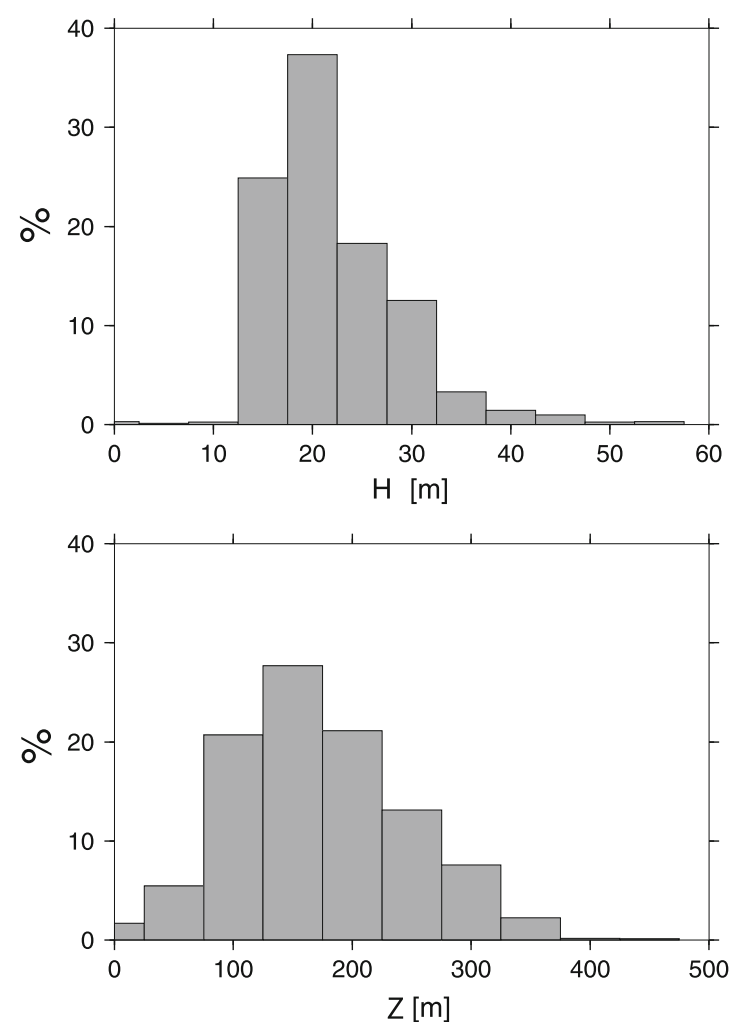

Fig. 5. The histogram of epicentral (up) and vertical (down) location errors for the analysed events. 
vertical uncertainties are partially due to multi-modality of the a posteriori distribution and thus are overestimated by the used estimator (covariance matrix). The dependence of the location errors upon a number of stations contributing to the hypocenter location is shown in Fig. 6 While the horizontal errors are almost independent of $N s$ (only a minor increase for small $N s$ can be observed) the vertical location errors significantly decreases for large $N s$. This is not surprising, because even for a planar seismic network a larger horizontal span of the contributing stations is efficiently enhancing the "vertical information" in data (Debski 1996, Debski et al. 1997) by differentiating the ratio of vertical-to-horizontal hypocenter-station distances.

Next, we have analysed the correlation between the hypocenter coordinates. They are shown in Fig. 7 where the off-diagonal elements of the a posteriori covariance matrix are shown.

The distinct feature visible in this figure is an existence of an overall small correlations (at the level of \pm 0.2 ) for most of events. Besides, we can see that for a number of events a large negative $R_{x y} \sim-0.4$ correlation between $x$ and $y$ coordinates has been reported. We interpret this as an effect of a particular (linear-like) station geometry for a given subset of events. Much more interesting is an existence of the secondary maximum for the $R_{x z}$ coefficients around the value $R_{x z} \sim-0.8$. This multi-modality of the $R_{x z}$ distribution suggests an existence of two classes of the solution. To verify this hypothesis we have analysed all 1D a posteriori marginal probability density distributions and we have found out that all solutions split generally into two classes. To the first class belong solutions for which the $P(z)$ distributions for the depth coordi-

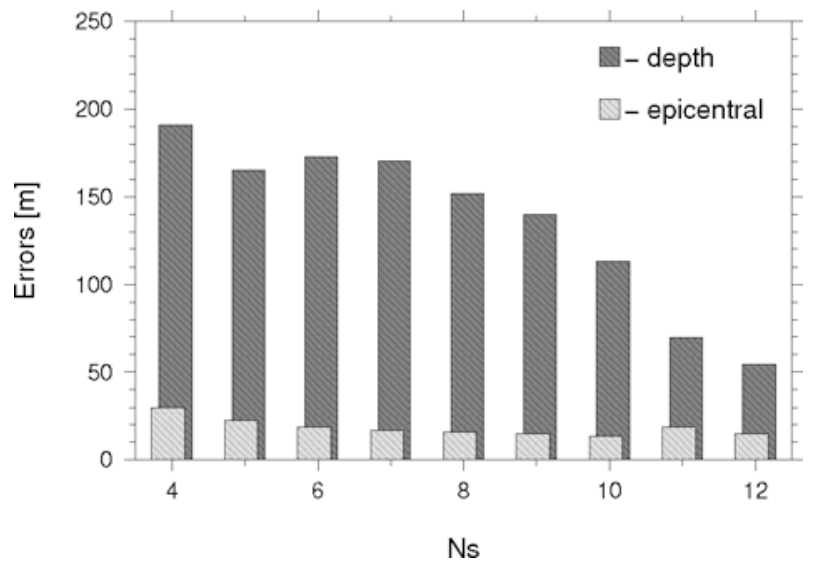

Fig. 6. The histogram of the epicentral and the vertical location errors upon the number of contributing seismic stations $N s$. While the horizontal errors are almost independent of $N s$ (only a minor increase for small $N s$ can be observed) the vertical location errors significantly decrease for large $N s$. 

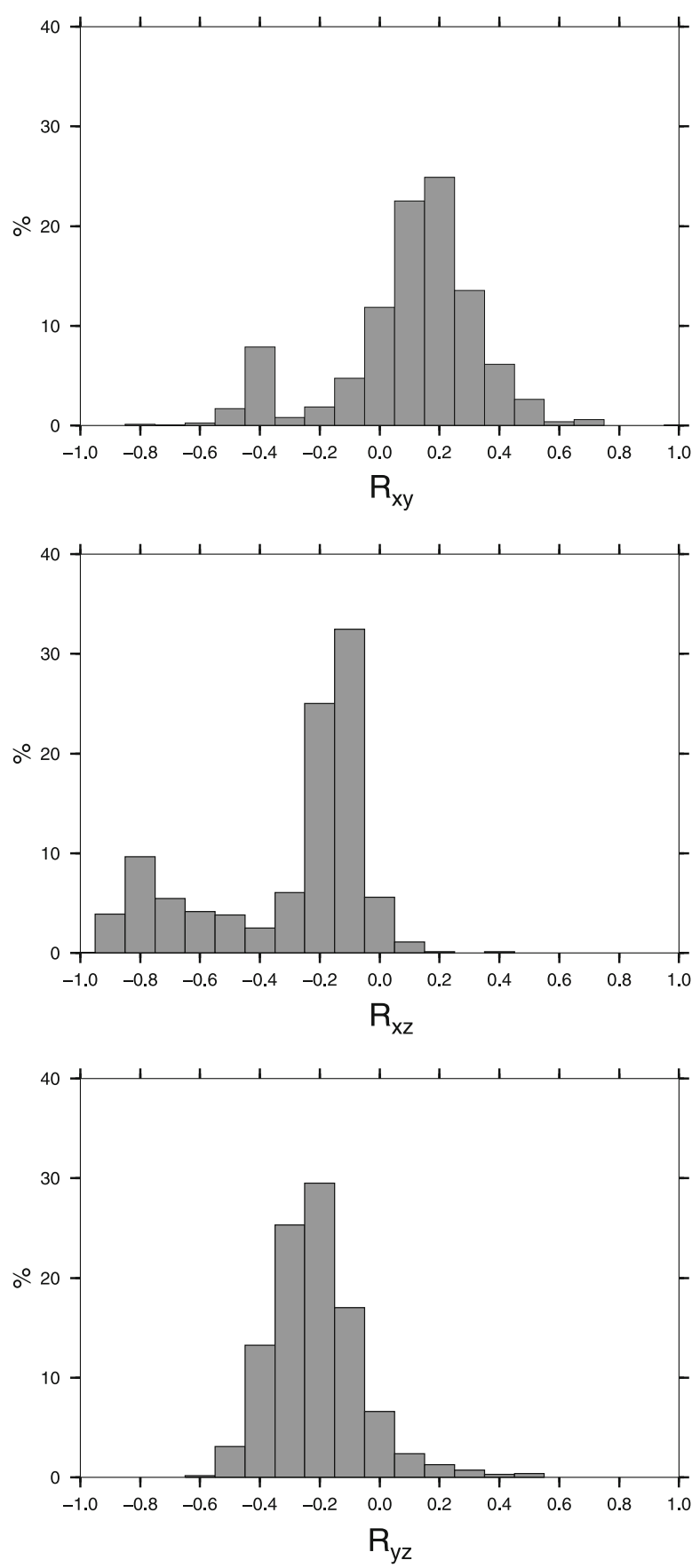

Fig. 7. The histogram of the correlation coefficients between hypocenter coordinates. In case of coefficients $R_{x y}$ and $R_{x z}$, the existence of two peaks is clearly visible. 

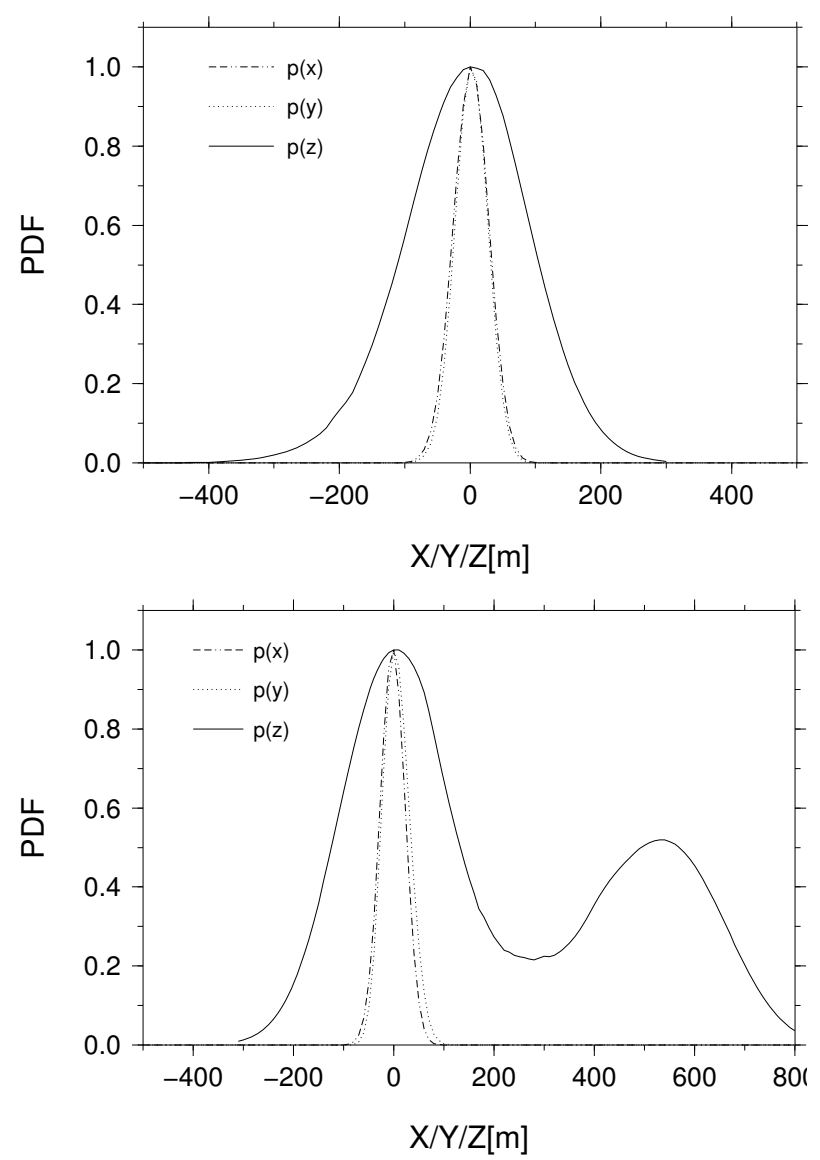

Fig. 8. The examples of two types of solutions with unimodal marginal depth distributions $P(z)$ (up) and two-modal distributions (down). While $P(x)$ and $P(y)$ distributions exibits typically a Gaussian-type shape for all events, the $P(z)$ splits into two class: unimodal (up) and two-modal (down). For the visualization purpose, all distributions were independently normalized and shifted towards unified maximum positions at zero.

nate are unimodal. The second class is formed by two-modal $P(z)$ distribution solutions. The distributions $P(z)$ typical for both classes are shown in Fig. 8

Similar 2D marginal distributions for exemplary events are shown in Fig. 9

The reason of the existence of two-modal depth solutions is obviously the planarity of the seismic underground network. For such a network geometry one can expect an existence of solution above and below the network plane. A possible remedy to this non-uniqueness problem (besides a rather costly hardware enhancing of the network) is using a more realistic, at least $1 \mathrm{D}$ velocity model differentiating between rock masses below and above the exploitation 

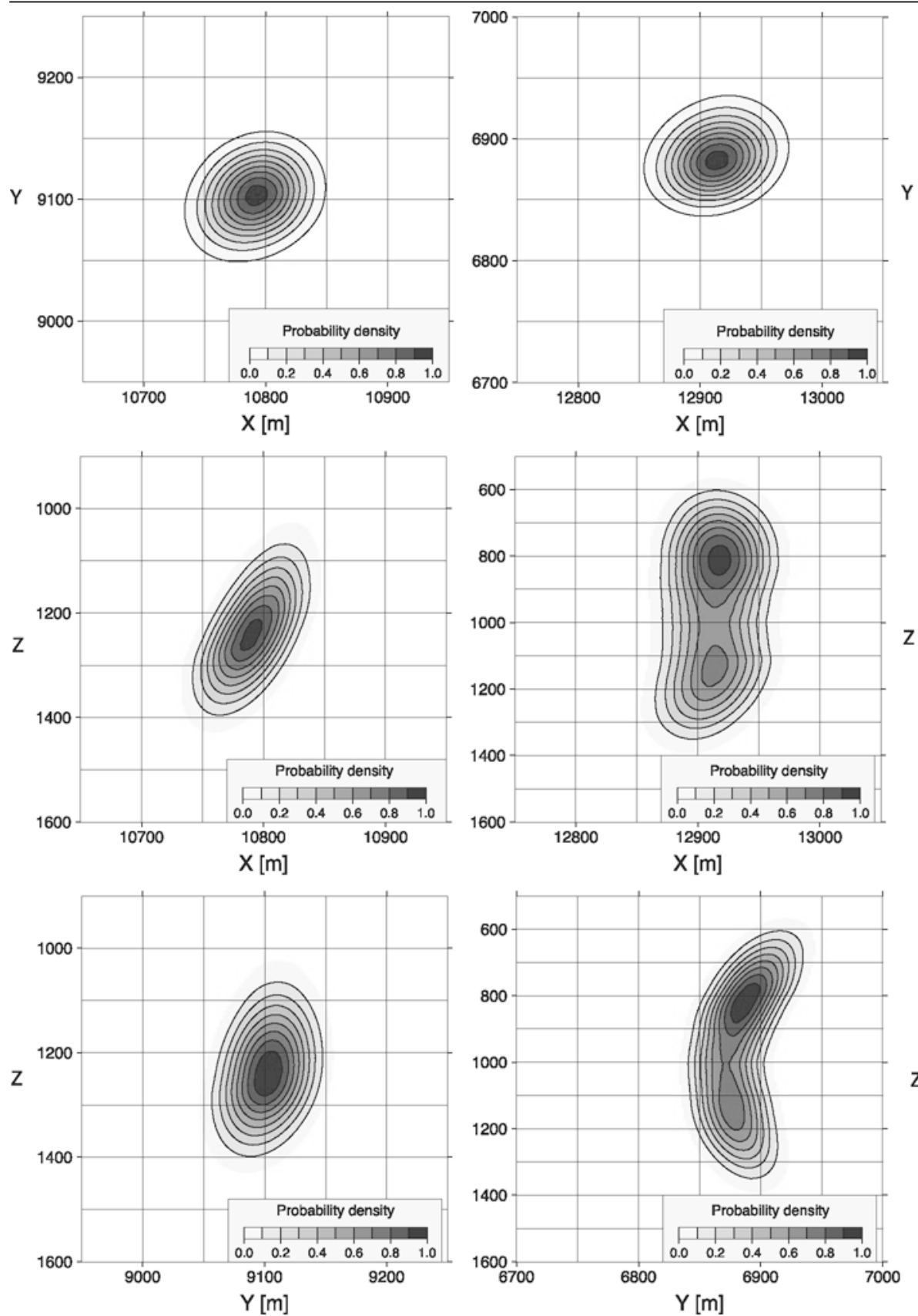

Fig. 9. The examples of two dimensional marginal distributions $P(x, y)$ (upper row), $P(x, z)$ (middle row), $P(y, z)$ (bottom row) for two classes of solutions: unimodal (left column) and two-modal (right column). Local coordinate system is used. 
level. The next conclusion following the existence of the multi-modal solutions is that for such a solution an error estimation by the covariance matrix is not justified (Debski 2010). In this case an error estimator "should measure" a width of the main pick only, otherwise, we get an overestimation of the location errors.

Finally, the question arises whether the secondary maxima appear systematically below or above the primary depth solutions. To answer this question we have calculated the skewness $\gamma$ coefficient for 1D a posteriori marginal distributions with respect to the position of the main pick. The results are shown in Fig. 10.
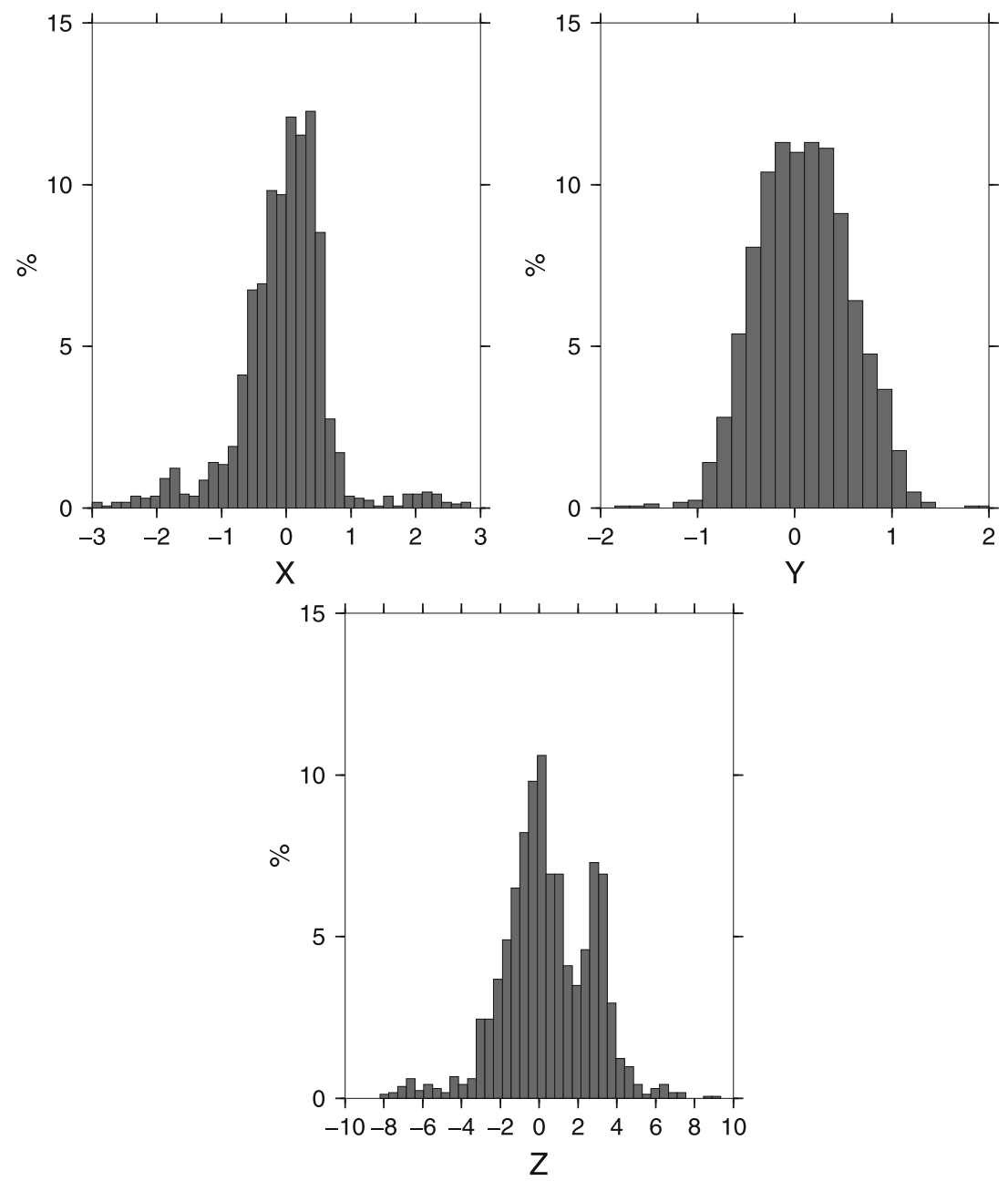

Fig. 10. The distribution of the skewness coefficients for the 1D a posteriori marginal distributions. 
The visible secondary pick in the skewness distribution of $P(z)$ occurs at the positive value of $\gamma$. It means that the secondary pick in two-modal solutions is more frequently localised at larger depths than the main solution.

\section{DISCUSSION AND CONCLUSIONS}

Evaluation of the quality of inversion results is an important element while solving any inverse task in hand. With the classical approach, when the solution is the optimum-fitting model, our ability of evaluation of inversion quality is very limited and relies in practice on linearized approach. Many statistical tests and methods have been developed along this line of reasoning (see, e.g., Brandt 1999). However, such an approach is more or less dependent on the particular solution found, so the results of the error analysis are "model dependent". The new possibilities are opened by the probabilistic approach which theoretically brings together all available uncertainties occurring during the inversion process and provides the statistics of the a posteriori errors. However, the approach is computationally demanding.

Exhaustive computations needed by the probabilistic inversion technique are due to two factors: a need of the sampling of the a posteriori distribution and the so-called "curse of dimensionality" effect (Curtis and Lomax 2001). The first factor is connected with the fact that obtaining some characteristics of the a posteriori distribution (average model, dispersion, etc.) requires inspection and evaluation of many models and thus many additional forward modelings. The second element, namely the fact that the models contributing to nonzero part of the a posteriori distribution forms a subset with a very small volume with respect to the whole model space makes the efficient sampling quite difficult (Mosegaard and Sambridge 2002). While building the TRMLOC algorithm we have explored the fact that for the source location task the model space (configuration space) is isomorphic with the space over which the forward operator (eikonal solver) is defined. This enabled us, by using the time reversal principle and the choice of the EDT-type likelihood function to make a calculation of the misfit function at all grid points having solved the forward problem for all receivers. In consequence there was no need of an additional sampling of the a posteriori pdf typical for classical Bayesian inversion. Obviously, a similar approach can be used in other situations when the model space coincides with the domain over which the forward operator solution is defined. For example, in case of the source location based on the full waveform inversion the misfit function can be defined by a difference between cross-correlations or more advanced measures (Kennett and Fichtner 2012) of the back propagated waveforms. The method can further be generalized by using different fields and different measures (Larmat et al. 2009, Ulrich et al. 2009) for constructing the misfit/likelihood function. The important question arises, however, at 
this point whether a similar method of "implicit sampling" can be developed for other time-reversal inverse problems when analyzed parameters are, for example source moment tensor, source time function, etc. An analysis of this issue will be presented elsewhere.

The important point of the developed algorithm is using the EDT type misfit function within the probabilistic inversion. The concept of the EDT misfit function is well established in seismology and its use within the classical, optimization-based inversion technique has proved to be very successful. However, using the EDT misfit to define the likelihood function in probabilistic inversion is by no means obvious. The reason is that the "true" likelihood function is actually the probability density function describing the sum of modelling and observational errors. Apparently, the likelihood-type function defined through the EDT misfit function does not have this property. The open question is thus if this difference is operationally important (eventually under which condition) or not. Apparently the maximum likelihood solution which is equivalent to the minimum of the misfit function is not influenced by this difference. However, this in not the case of other moments, like for example variance of the a posteriori distribution and thus some systematic bias can occur. This issue apparently must be clarified.

Finally, let us note that the seismic source location task is a very special type of inverse problem in which the model space is the same as the space over which the forward operator acts. We have explored this identity in the TRMLOC algorithm to perform the implicit sampling of the a posteriori distribution and thus ensuring its very high numerical efficiency. We think that this approach, when combined with time-reversal principle, can further be extend to analyze different characteristics of seismic sources. The crucial point is the observation that the seismic signals back-propagated to the hypocenter location contain all information about the source as the originally recorded waveforms. Thus, to perform the full waveform inversion for seismic source parameters we do not need to use the originally recorded waveforms. Instead we can use the back-in-time propagated signals at the hypocenter location. In consequence, the most time consuming part of any full waveform inversion, namely the propagation of seismic waves from hypocenter to recording stations performed many times during inversion can be replaced by a single back-in-time seismic waves propagation. Apparently, additional uncertainties are introduced with such an approach and a lot of further analysis is necessary.

The developed algorithm has allowed us to make a nontrivial error analysis for a set of 1647 events from Rudna copper mine. The most interesting result of this analysis is demonstrating of an existence of a sub-class of two-modal solutions. We have found out that in most cases the secondary maximum in the a posteriori distributions occur at larger depths (often below the exploitation level). Possibilities of such deeper, below the exploitation level, seismic 
events were discussed, for example, by Gibowicz and Kijko (1994), Gibowicz and Lasocki (2001). In the light of the obtained results, a hypothesis of generation of seismic events in the footwall in Rudna copper mine cannot be ruled out.

Acknowledgment. This paper was partially support by grant No. 2011/01/B/ST10/07305 from the National Science Center, Poland. G. O'Brien and the anonymous reviewers are acknowledged for their help in improving the manuscript.

\section{References}

Aki, K., and P. Richards (1985), Quantitative Seismology, Freeman and Co., San Francisco

Artman, B., I. Podladtchikov, and B. Witten (2010), Source location using timerevers imaging, Geophys. Prosp. 58, 5, 861-873, DOI:10.1111/j.13652478.2010.00911.x

Bai, L., Z. Wu, T. Zhang, and I. Kawasaki (2006), The effect of distribution of stations upon location error: Statistical tests based on the double-difference earthquake location algorithm and the bootstrap method. Earth Planets Space 58, 2, e9e12, DOI:10.1186/BF03353364.

Brandt, S. (1999), Data Analysis. Statistical and Computational Methods for Scientists, Springer-Verlag.

Bulland, R. (1976), The Mechanics of locating earthquakes, Bull. Seismol. Soc. Am. 66, 1, 173-187.

Chib, S. and Greenberg (1995), Understanding the Metropolis-Hastings Algorithm, Am. Stat. 49, 327-335, DOI: 10.1080/00031305.1995.10476177.

Curtis, A. and A. Lomax (2001), Prior information sampling distributions and the curse of dimensionality. Geophysics 66, 2, 372-378, DOI:10.1190/1.1444928.

Dagum, L. and R. Menon (2002), OpenMP: an industry standard API for sharedmemory programming, Comput. Sci. Eng. IEEE 5, 1, 46-55, DOI:10.1109/ 99.660313 .

Debski, W. (1996). Location of Seismic Events A Quest for Accuracy, SpringerVerlag, Berlin, DOI: 10.1007/BFb0011773.

Debski, W. (2004). Application of Monte Carlo techniques for solving selected seismological inverse problems, Publs. Inst. Geophys. Pol. Acad. Sc. B-34, 367, $1-207$.

Debski, W. (2010), Probabilistic inverse theory, Adv. Geophys. 52, 1-102, DOI:10.1016/S0065-2687(10)52001-6. 
Debski, W. (2015), Using meta-information of a posteriori Bayesian solutions of the hypocenter location task for improving accurcy of location error estimation, Geophys. J. Int. 201, 3, 1399-1408, DOI:1093/gji/ggv083.

Debski, W., B. Guterch, H. Lewandowska, and P. Labak (1997), Earthquake sequences in the Krynica region Western Carpathians 1992 - 1993, Acta Geophys. Pol. XLV, 4, 255-290.

Fink, M. (1997), Time reversed acoustic, Physics Today 50, 3, 34-40, DOI:10.1063/1.881692.

Fink, M., D. Cassereau, A. Derode, C. Prada, P. Roux, M. Tanter, J.-L. Thomas, and F. Wu (2000), Time-reversed acoustics, Reports on Progress in Physics 63, 12, 1933-1994.

Fink, M. and M. Tanter (2010), Multiwave imaging and super resolution. Physics Today 63, 2, 28-33, DOI:10.1063/1.3326986.

Font, Y., H. Kao, S. Lallemand, C.-S. Liu, and L.-Y. Chiao (2004), Hypocentre determination offshore of eastern Taiwan using the Maximum Intersection method, Geophys. J. Int. 158, 655-675, DOI:10.1111/j.1365-246X.2004.02317.x.

Gajewski, D. and E. Tessmer (2010), Reverse modelling for seismic event characterization, Geophys. J. Int. 163, 1, 276-284, DOI: 10.1111/j.1365246X.2005.02732.x.

Gibowicz, S. J. and A. Kijko (1994), An Introduction to Mining Seismology. San Diego: Academic Press.

Gibowicz, S. J. and S. Lasocki (2001), Seismicity Induced by Mining: Ten Years Later. Adv. Geophys. 44, 39-181, DOI:10.1016/S0065-2687(00)80007-2.

Gilks, W., S. Richardson, and D. Spiegelhalter (1995), Markov Chain Monte Carlo in Practice, Chapman\& Hall/CRC Press.

Giovambattista, R. and S. Barba (1997), An estimate of hypocentre location accuracy in a large network: possible implications for tectonic studies in Italy, Geophys. J. Int. 129, 1, 124-132, DOI:10.1111/j.1365-246X.1997.tb00941.x.

Hauser, J., M. Sambridge, and N. Rawlinson (2008). Multiarrival wavefront tracking and its applications, Geochem. Geophys., Geosys. 9, 11, DOI:10.1111/j.1365246X.1997.tb00941.x.

Husen, S. and J. Hardebeck (2010). Earthquake location accuracy, Community online resources for statistical seismicity analysis. DOI: 10.5078/corssa-55815573.

Husen, S., E. Kissling, E. Flueh, and G. Asch (1999), Accurate hypocentre determination in the seismogenic zone of the subducting Nazca Plate in northern Chile using a combined on-/offshore network, Geophys. J. Int. 138, 3, 687-701, DOI:10.1046/j.1365-246x.1999.00893.x.

Kennett, B. and A. Fichtner (2012), A unified concept for comparison of seismograms using transfer functions, GJI 191, 3, 1403-1416, DOI: 10.1111/j.1365246X.2012.05693.x. 
Klein, F. (2002), User's guide to HYPOINVERSE-2000: A Fortran program to solve for earthquake locations and magnitudes, US Geological Survey.

Kloc, M. and T. Danek (2012), The Multi GPU Accelerated Waveform Inversion in Distributed OpenCL Environment, Volume 151 of Lecture Notes in Electrical Engineering, Springer New York, .

Kremers, S., A. Fichtner, G. Brietzke, H. Igel, C. Larmat, L. Huang, and M. Kaser (2011), Exploring the potentials and limitations of the time-reversal imaging of finite seismic sources. Solid Earth 2, 1, 95-105, DOI:10.5194/se-2-95-2011.

Lahr, J. (1989), HYPOELIPSE (revised); A computer program for determining local earthquake hypocentral parameters, magnitude and first motion pattern, US Geological Survey.

Larmat, C., R. Guyer, and P. A. Johnson1 (2009), Tremor source location using time reversal: Selecting the appropriate imaging field, Geophys. Res. Lett. 36, (L22304),DOI:10.1029/2009GL040099 .

Larmat, C., J. Tromp, Q. Liu, and J.-P. Montagner (2008), Time reversal location of glacial earthquakes, J. Geophys. Res. 113, B09314, 1-9, DOI:10.1029/2008JB005607

Lee, W. and J. Lahr (1975), HYPO71 (revised); A computer program for determining hypocenter, magnitude, and first motion pattern of local earthquakes, US Geological Survey.

Lehmann, E. L. and G. Casella (1998). Theory of Point Estimation, Springer Texts in Statistics. New York: Springer-Verlag.

Lomax, A. (2005). A Reanalysis of the Hypocentral Location and Related Observations for the Great 1906 California Earthquake, Bull. Seismol. Soc. Am. 95, 3, 861877, DOI: 10.1785/0120040141.

Lomax, A., A. Michelini, and A. Curtis (2009), Earthquake Location, Direct, GlobalSearch Methods, Volume 5. New York: Springer, DOI: 10.1007/978-0-38730440-3.

Lomax, A., J. Virieux, P. Volant, and C. Berge (2000). Probabilistic earthquake location in 3D and layered models: Introduction of a Metropolis-Gibbs method and comparison with linear locations, Amsterdam: Kluver, DOI:10.1007/97894-015-9536-0_5.

Lomax, A., A. Zollo, P. Capunao, and J. Virieux (2001), Precise absolute earthquake location under Somma-Vesuvius volcano using a new three-dimensional velocity model, Geophys. J. Int. 146, 2, 313-331, DOI:10.1046/j.0956540x.2001.01444.x.

Masson, Y., P. Cupillard, Y. Capdeville, and B. Romanowicz (2014), On the numerical implementation of time-reversal mirrors for tomographic imaging. Geophys. J. Int., 3, 1-11, DOI:10.1093/gji/ggt459. 
Matsu'ura, M. (1984), Bayesian estimation of hypocenter with origin time eliminated, J. Phys. Earth. 32, 6, 469-483.

Menke, W. (1989), Geophysical Data Analysis: Discrete Inverse Theory, International Geophysics Series. San Diego: Academic Press.

Mosegaard, K. and M. Sambridge (2002). Monte Carlo analysis of invers problems. Inv. Prob. 18, 3, R29-45, DOI: 10.1088/0266-5611/18/3/201.

Mosegaard, K. and A. Tarantola (2002), International Handbook of Earthquake \& Engineering Seismology, Volume 81 of International Geophysics Series. Academic Press.

O`Brien, G., J. Lokmer, L. D. Barros, C. Bean, G. Saccorotti, J.-P. Metaxian, and D. Patane (2011). Time reverse location of seismic long-period events recorded on Mt Etna. Geophys. J. Int. 184, 1, 452-462, DOI:10.1111/j.1365246X.2010.04851.x.

Pavlis, G. L. (1992), Appraising relative earthquake location errors, Bull. Seismol. Soc. Am. 82, 2, 836-859.

Podvin, P. and I. Lacomte (1991), Finite-difference computtion of traveltimes in very contrasted velocity models: a massively paarallel approach and its associated tools, Geophys. J. Int. 105, 1, 271-284, DOI:10.1111/j.1365246X.1991.tb03461.x.

Quinn, M. (2008), Parallel Programming in C with MPI and OpenMP. McGraw-Hill Education.

Rawlinson, N. and M. Sambridge (2004), Multiple reflection and transmission phases in complex layered media using a multistage fast marching method. Geophys. 69 , 5, 2178-2193, DOI:10.1190/1.1801950.

Rudzinski, L. and W. Debski (2011). Extending the Double Difference location technique for mining applications - part I: Numerical study. Acta Geophys. 59, 4, 785-814, DOI:10.2478/s11600-011-0021-5.

Rudzinski, L. and W. Debski (2012), Extending the Double Difference location technique - improving hypocenter depth determination. J. Seismol. 17, 1, 83-94, DOI:10.1007/s10950-012-9322-7.

Sambridge, M. and K. Mosegaard (2002), Monte Carlo Methods in Geophysical Inverse Problems. Rev. Geophys. 40, 3, 3.1-3.29, DOI:10.1029/2000RG000089.

Scalerandi, M., M. Griffa, and P. Johnson (2009), Robustness of computational time reversal imaging in media with elastic constant uncertainties. J. Appl. Phys. 106, 114911, DOI:10.1063/1.3269718.

Sethian, J. A. (1999), Level set methods and fast marching methods: evolving interfaces in computational geometry fluid mechanics computer vision and materials science. Cambridge Monographs on Applied and Computational Mathematics. New York: Cambridge University Press. 
Steiner, B. and E. Saenger (2012), Comparison of 2D and 3D time-reverse imaging - A numerical case study, Comput \& Geosci. 46, 174-182, DOI:10.1016/j.cageo.2011.12.005.

Tarantola, A. (2005), Inverse Problem Theory and Methods for Model Parameter Estimation, Philadelphia: SIAM.

Thurber, C. and N. Rabinowitz (2000). Advances in Seismic Event Location, Volume 18. Springer.

Tromp, J., C. Tape, and Q. Liu (2005), Seismic tomography, adjoints methods, time reversal and banana-doughnut kernels, Geophys. J. Int. 160, 1, 195-216, DOI:10.1111/j.1365-246X.2004.02453.x.

Ulrich, T., K. V. D. Abeele, P.-Y. L. Bas, M. Griffa, B. Anderson, and R. Guyer (2009), Three component time reversal: Focusing vector components using a scalar source, J. Appl. Physics 106, 11, 113504, DOI:10.1063/1.3259371.

Ulrich, T., A. Sutin, R. Guyer, and P. Johnson (2008), Time reversal and non-linear elastic wave spectroscopy (TR NEWS) techniques, Int. J. of Non-Lin. Mech. 43, 3, 209-216, DOI:10.1016/j.ijnonlinmec.2007.12.017.

Vidale, J. (1990), Finite-difference calculation of traveltime in three dimensions. Geophysics 55, 5, 521-526, DOI:10.1190/1.1442863.

Virieux, J., S. Operto, H. Ben-Hadj-Ali, R. Brossier, V. Etienne, and F. Sourbier (2009). Seismic wave modeling for seismic imaging. Leading Eadge,28, 5, 538-544, DOI:10.1190/1.3124928.

Waldhauser, F. and W. Ellsworth (2000), A double-difference earthquake location algorithm: method and application. Bull. Seismol. Soc. Am. 90, 6, 1353-1368.

Wiejacz, P. and W. Debski (2001), New Observation of Gulf of Gdansk Seismic Events. Phys. Earth Planet. Int. 123, 2-4, 233-245, DOI:10.1016/S00319201(00)00212-0.

Witten, B. and B. Artman (2011). Signal-to-noise estimates of time-reverse images. Geophysics 76,2, MA1-MA10, DOI:10.1190/1.3543570.

Zhang, L., J. Rector, and G. Hoversten (2005), Eikonal solver in the celerity domain, Geophys. J. Int. 162, 1, 1-8, DOI:10.1111/j.1365-246X.2005.02626.x.

Zhao, H. (2005), Fast Sweeping Method for Eikonal equations. Math. Comput. 74, 603-627, DOI:10.1090/S0025-5718-04-01678-3.

Zhou, H. (1994), Rapid three-dimensional hypocentral determination using a master station method, J. Geophys. Res. 99, B8, 715439-15455, DOI:10.1029/94JB00934.

Received 19 February 2015 Received in revised form 29 October 2015

Accepted 19 November 2015 